\title{
Análise da complexidade, estratégias e aprendizagem em projetos de melhoria contínua: estudos de caso em empresas brasileiras
}

\author{
Analysis of complexity, strategies, and learning \\ organization in continuous improvement processes: \\ case studies in brazilian companies
}

\author{
Pedro Carlos Oprime ${ }^{1}$ \\ Rafael Monsanto ${ }^{1}$ \\ Júlio Cesar Donadone ${ }^{1}$
}

\begin{abstract}
Resumo: O objetivo deste artigo é analisar projetos de melhoria contínua desenvolvidos por empresas industriais brasileiras com base na abordagem/programa da Manutenção Produtiva Total (Total Productive Maintenance, TPM) e da Qualidade Total (Total Quality Management, TQM). As variáveis estudadas nas análises dos projetos foram as seguintes: i) complexidade dos projetos; ii) seus objetivos específicos; iii) as estratégias competitivas relacionadas; iv) o aprendizado organizacional obtido; e v) as ferramentas e os principais pilares do TPM implementados. Selecionou-se para estudo projetos considerados de sucesso por suas empresas. Ao todo, analisaram-se 42 projetos de 12 diferentes empresas que representaram diversos segmentos industriais do Brasil. Os resultados indicaram correspondência entre a natureza do projeto, em termos da sua complexidade e estrutura organizacional, com a estratégia competitiva adotada.
\end{abstract}

Palavras-chave: Qualidade. Estratégia. Eficiência. Produção. TPM. Aprendizado.

\begin{abstract}
This paper analyses the major goals and results of TPM and TQM processes in companies that adopt these approaches. The main variables considered in this study were: $i)$ complexity of the projects developed by the companies; ii) projects' specific goals iii) relationship between the projects and the company's strategy; iv) relationship between projects and continuous improvement and organizational learning; $v$ ) tools and TPM concepts that support the projects. A descriptive research was carried out using field research strategies. Forty-two processes adopted by 12 companies were analyzed in this study. The processes implemented were considered successful by the companies that adopt them and by outside specialists. The results indicated close relationship between the project's nature (its complexity and team structure) and the company`s strategy, project goals and organizational learning.
\end{abstract}

Keywords: Quality. Strategy. Efficiency. Production. TPM. Learning.

\section{Introdução}

Enquanto o diferencial competitivo de uma empresa é tradicionalmente atribuído à qualidade do produto (GARVIN,1993), mais recentemente as organizações vêm experimentando preocupações no que concernem às mudanças da percepção de valor atribuído pelos clientes (SUPANVANIJ; AMINE, 2000). Em tese, o ganho econômico direto, somente focado na eficiência da organização, não é a única razão para a busca da melhoria contínua de produtos e processos, pois a estratégia da produção se diversificou nas últimas décadas, incluindo à dimensão custos a qualidade, a velocidade de entrega, o tempo/agilidade, a flexibilidade e a inovação de produtos e serviços (CHASE; JACOBS; AQUILANO, 2006; SLACK et al., 2007). Entretanto, as demandas, representadas por uma ampla gama de atores, apresentam-se variadas e complexas, induzido as empresas a incorporarem na sua gestão temas como a responsabilidade social, a ética e a preocupação com o impacto ambiental e têm fomentado discussões sobre a revisão do papel e das atuais abordagens da gestão da qualidade total.

Tendo como ponto de partida o fato de que as empresas têm priorizado ganhos de produtividade/ eficiência nas suas operações, decorrente do acirramento

\footnotetext{
${ }^{1}$ Departamento de Engenharia de Produção, Universidade Federal de São Carlos - UFSCar, São Carlos - SP, Brasil,

E-mails: pedro@dep.ufscar.br; monsanto.rafael@gmail.com; julio@ dep.ufscar.br
}

Recebido em 13/5/2009 — Aceito em 24/8/2010

Suporte financeiro: CNPq. 
da competitividade, vê-se que a melhoria contínua ocupa um espaço significativo na gestão das empresas. A princípio, a melhoria contínua caracteriza-se por promover, por meio de atividades de grupos, pequenas melhorias em produtos e processos, o que pode nesse momento não ser a resposta suficiente para enfrentar os novos desafios pelas empresas. Como um conceito em evolução, a melhoria tende à inovação contínua, que propõe mudanças mais radicais pela introdução de novas tecnologias e pela incorporação de novos procedimentos, métodos, estruturas administrativas e processos aos padrões atuais das empresas (PRAJOGO; SOHAL, 2001; NILSSON-WITELL; ANTONI; DAHLGAARD, 2005; UPTON, 1998).

Com o propósito de buscar as novas tendências da gestão da qualidade, por ser um dos elementos centrais das práticas gerenciais, procurou-se neste trabalho analisar as relações entre as atuais práticas de Melhoria Contínua (MC) com a estratégia e aprendizado organizacional.

A pergunta a ser respondida é: como a melhoria contínua tem contribuído no atendimento aos objetivos estratégicos e no aprendizado organizacional? A proposição é que as atividades de melhoria contínua tornaram-se, ao longo das últimas décadas, um elemento estratégico para as empresas, propiciando a incorporação de inovações e o aprendizado à organização. $O$ foco deste trabalho são os projetos de melhoria contínua desenvolvidos por empresas que adotam alguma abordagem/programa/método para a melhoria contínua, em especial o TQM (Total Quality Mangement) e TPM (Total Productive Maintenance).

Há autores que afirmam que a qualidade total (TQM) na administração da produção tem representado um papel estratégico e contribuído para a competitividade de muitos setores e segmentos industriais, por exemplo, nos setores automobilístico e de serviços (PRAJOGO; SOHAL, 2001; RAHMAN; BULLOCK, 2005). Isso se explica em parte pelo fato da gestão da qualidade total ter como foco a melhoria contínua de produtos e processos e as necessidades dos clientes. Os conceitos, técnicas e ferramentas do TQM e os modelos de gestão da qualidade, tais como a ISO 9000:2008, já estão bastante difundidos no meio industrial, porém há autores que argumentam que o TQM é pouco pragmático em relação a outras abordagens, tal como a do Seis Sigma (EHIGIE; McANDREW, 2005). Outros autores apresentam argumentos que indicam a renovação do TQM por meio da inserção de novas abordagens/métodos, como, por exemplo, o Seis Sigma (NILSSON-WITELL; ANTONI; DAHLGAARD, 2005; FOSTER; JONKER, 2007).

Como elemento central do TQM, a melhoria contínua é dinâmica nos seus conceitos e modos de organizar suas atividades, especificamente na organização dos grupos de melhoria e nas estratégias de suporte e incentivos dados aos grupos. Novas abordagens têm sido introduzidas pelo Seis Sigma e Manutenção Produtiva Total (TPM), que estabelecem uma forma de organização e operacionalização das atividades de melhoria que melhor respondem às necessidades estratégicas das empresas.

Isso se explica, quando se observa a história da MC no início do século XX, no contexto da chamada administração científica cunhada por Ford e Taylor, que ela tinha como foco a eficiência da produção por meio da racionalização dos processos das indústrias com o objetivo de reduzir custos. Não deixando de lado a racionalização dos processos, as empresas têm no século XXI o desafio de atender a novas demandas dos diversos agentes envolvidos com ela, representados pelos clientes, governos, entidades de classe e comunidade local. Isso abre espaço para novas formas de organizar e operacionalizar a melhoria contínua e o TQM, como constata Ehigie e McAndrew (2005) que fizeram um estudo das publicações sobre o TQM, mostrando que o auge de suas publicações ocorreu por volta do início dos anos noventa e que teve queda significativa no final da mesma década. A argumentação dos autores é que programas como o Seis Sigma e TPM, por serem mais rígidos e formais, têm levado as empresas que os adotaram a obterem resultados satisfatórios (EHIGIE; McANDREW, 2005; BERGQUIST; FREDRIKSSON; SVENSSON, 2005; McADAM; HENDERSON, 2004; LEONARD; McADAM, 2003).

Outro programa relacionado à melhoria contínua bastante difundido nas empresas é o TPM, de origem japonesa, que surgiu por volta de 1971, e que tem como base o PM (Manutenção Preventiva). Esse programa foi introduzido nos EUA nos anos 50 e 60. Subsequentemente, nos anos 70 e até os anos 80 , o TPM foi se desenvolvendo gradualmente e passou a ser um dos programas mais importantes aplicados pela indústria no mundo (SHIROSE; BODEK, 1992).

Tendo em conta a importância da melhoria contínua para o desenvolvimento das habilidades e competências das organizações e o fato desta ser o foco de diferentes programas/abordagens (tais como TQM, Seis Sigma e TPM), é objetivo desta pesquisa mostrar que as atividades de melhoria contínua têm tido um enfoque estratégico e menos operacional, diferentemente do que ocorreu na origem desses programas. Para atender aos objetivos e responder à questão de pesquisa, foram analisados 42 projetos de MC considerados os mais relevantes pelas empresas e que foram pré-selecionados pelos autores desse artigo. Para dar suporte teórico à escolha das variáveis e nas análises dos resultados, foi realizada uma revisão da literatura sobre TQM, melhoria contínua e TPM. Nas análises dos projetos, foram consideradas as variáveis relacionadas aos seguintes aspectos: i) a natureza dos projetos desenvolvidos pelas empresas; ii) os objetivos específicos dos projetos; iii) as relações desses 
projetos com os objetivos estratégicos das empresas; iv) relação entre a melhoria contínua e aprendizado organizacional; e v) as principais ferramentas e os principais pilares do TPM que suporta os projetos.

A população de interesse são os projetos de melhoria com impactos estratégicos para as empresas que os desenvolveram, e o tipo de amostragem aplicada foi a intencional, pois o objetivo é estabelecer conclusões sobre a população a partir da amostra. Assim, buscou-se obter sua representatividade pela seleção dos melhores projetos de melhoria.

Para alcançarmos os objetivos propostos, este artigo está estruturado em seis seções, incluindo as referências bibliográficas. Na primeira seção delineia-se o escopo do trabalho, sua justificativa e seus objetivos. Na segunda seção, sintetizam-se aspectos teóricos relacionados à temática de pesquisa, para, em seguida, apresentar o procedimento metodológico. $\mathrm{Na}$ quarta seção, sumarizam-se os resultados e análise dos dados; em seguida estão as conclusões finais do trabalho.

\section{Revisão da literatura}

\subsection{Abordagens de melhoria contínua}

Há uma considerável bibliografia sobre o TQM, na qual seus aspectos mais importantes são tratados. Alguns autores como Merli (1993), Juran e Gryna (1991), Garvin, (1993) e Toledo (1987) fornecem uma ampla visão dos vários aspectos que o envolvem, por exemplo, conceitos e definições, o papel da liderança da alta administração, ferramentas e métodos, bem como a importância da cultura organizacional para a qualidade.

Pode-se dizer que os atuais programas, sistemas da qualidade e modelos (tais como a ISO 9000:2000, os prêmios nacionais da qualidade e Seis Sigma) fazem parte da evolução do TQM (CUSUMANO, 1994).

O futuro do TQM é um tanto incerto, porém Nilsson-Witell, Antoni e Dahlgaard (2005) e Foster e Jonker (2007) avaliam o desenvolvimento do TQM e fornecem indícios de uma nova abordagem para a gestão da qualidade. Essa abordagem incorpora novos objetivos, que são mais estratégicos, voltados a questões críticas para a manutenção e perpetuação dos negócios, como, por exemplo, a sustentabilidade e o meio ambiente, o bem estar social e compromisso e transparência nas relações com a comunidade local, com os clientes e governos.

Autores como Ehigie e McAndrew (2005) questionam se o TQM é um conceito enraizado ou uma prática em desuso. Para ilustrar a discussão sobre o assunto, eles tomaram o caso dos círculos de controle da qualidade (CCQ) que foram muito estudados e implementados no final da década de 80, mas nas décadas seguintes saíram do foco da discussão teórica e prática. Para os autores, o mesmo tem ocorrido com o TQM, que saiu do foco principal dos estudos e pesquisas, por conta da entrada de novos programas, tais como o Seis Sigma e TPM.

Poder-se-ia dizer que novas abordagens do TQM têm surgido como o Seis Sigma, enquanto outras têm caído em desuso, em geral devido à falta de resultados práticos da aplicação ou pela incorporação de novos conceitos (EHIGIE; McANDREW, 2005; BERGQUIST; FREDRIKSSON; SVENSSON, 2005; McADAM; HENDERSON, 2004; LEONARD; McADAM, 2003; RAISINGHANI et al., 2005). Apesar disso, há autores, como Prajogo e Sohal (2004), que argumentam que o movimento da qualidade tem contribuído de forma positiva para o desempenho das empresas, pois o TQM é ainda um elemento estratégico para empresas competirem por diferenciação de produtos e serviços. Tendo em conta essa argumentação, parece ainda que a qualidade é um fator ganhador de pedidos (SLACK et al., 2007), entretanto essa questão é mais complexa, pois, para muitos setores, qualidade é um fator qualificador para a entrada no mercado.

O que se tem percebido é que conceitos clássicos do TQM ainda têm se difundido em organizações típicas de serviços e também do setor público (LEONARD; McADAM, 2003; McADAM; HENDERSON, 2004). Isso indica que há ainda espaço para a difusão do TQM nos moldes conhecidos no seu primórdio. Porém, analisar o TQM somente por esse aspecto não fornece elementos que demonstrem sua importância perante as organizações. Há outros programas, estruturas e pragmáticos nos resultados, como o Seis Sigma e o TPM, que têm no seu escopo como elemento central a melhoria contínua e grande parte da filosofia, técnicas e ferramentas da qualidade.

Diferentes aspectos da Melhoria Contínua (MC) têm sido publicados, a começar pela sua história, cujos primeiros registros apontam já existirem atividades de melhoria contínua, ainda que informais e desestruturadas, na indústria americana do final do século XIX. Programas formais aparecem pela primeira vez no Japão, na segunda metade do século XX (JHA; NOORI; MICHELA, 1996; BHUIYAN; BAGHEL, 2005). Ocorre, a partir de então, grande disseminação de programas de melhoria contínua nas empresas no Brasil e no mundo. Isso se deve em parte à modernização das práticas gerenciais, em que as relações humanas no trabalho passam a ser incorporadas à rotina das empresas. Há ainda o acirramento da concorrência e, por conseguinte, da competitividade, gerando pressão por desempenhos em produtividade, melhor qualidade dos produtos e serviços, flexibilidade de volume e de produção, rapidez no atendimento aos clientes e inovações dos produtos (SLACK et al., 2007). Comparados com as décadas passadas, os desafios atuais enfrentados pelas empresas são mais 
complexos, exigem maiores competências técnicas e habilidades gerenciais, bem como a utilização mais eficiente de técnicas e ferramentas na identificação e solução de problemas (BESSANT et al., 1994; BESSANT; CAFFYN, 1997; BESSANT, 1999; BESSANT; CAFFYN; GALLAGHER, 2001).

Nesse contexto, a melhoria contínua de produtos e processos desempenha um papel ainda mais importante para a competitividade. Porém, a implementação da melhoria contínua envolve a renovação organizacional em termos não só culturais, mas na forma como as atividades ocorrem nas organizações. Isso significa introduzir novos comportamentos e reformas na estrutura administrativa, em especial ideológica e gerencial (SAVOLAINEN, 1999). A estrutura antiga, centralizada e autocrática, não propicia a agilidade e mudanças, e é preciso, nos dias atuais, uma renovação de valores e posturas que envolvem mudanças nas práticas organizacionais.

A literatura apresenta um conjunto significativo de fatores que direta ou indiretamente tem relações com o processo de melhoria contínua, por exemplo: os métodos de controle das atividades dos grupos de melhoria (DABHILKAR; BENGTSSON, 2004); os mecanismos de cooperação lateral (HAMMERSLEY; PINNGTON, 1999; JABNOUN, 2001; LEE, 2004; ABRAHAMSSON; GERDIN, 2006); o compartilhamento de experiências e aprendizados (BESSANT; FRANCIS, 1999; BESSANT; CAFFYN; GALLAGHER, 2001); as práticas gerenciais e o processo de renovação organizacional (HARRINGTON, 1995; SAVOLAINEN, 1999); a relação entre a melhoria contínua e os sistemas de gestão da qualidade, tal como a ISO 9000 (SACCHETTI, 2007; TERZIOVSKI; POWER, 2007); a relação entre melhoria contínua, inovação contínua e aprendizagem organizacional (BOER; GERTSEN, 2003); os mecanismos de suporte e uso de ferramentas para a melhoria contínua (BESSANT et al., 1994; CHAPMAN; HYLAND, 1997; HYLAND et al., 2000; JOINER, 2007); o grau de participação dos indivíduos frente a diferentes modos de organização e suporte às atividades de melhoria (DELBRIDGE; BARTON, 2002); as formas de controle das atividades de MC e suas implicações (DABHILKAR; BENGTSSON, 2004); o uso de indicadores de desempenho e de sistemas de informação para o suporte às atividades de melhoria (BECKET; WAINWRIGHT; BANCE, 2000; BOND, 1999); e as diferenças e implicações das organizações do tipo top-down e bottom-up para a melhoria contínua (HARRINGTON, 1995; IMAI, 1997; TERZIOVSKI, 2002).

Não é tarefa fácil implementar mudanças de valores e de paradigmas. O processo de mudança de valores e paradigmas tem que ser feito pelo líder ou induzido por uma lenta mudança comportamental planejada, de modo racional e controlada. As mudanças desejadas de níveis de maturidade no comportamento e das práticas da melhoria contínua em uma organização só têm chance de ocorrer se houver apoio e a participação da alta gerência (SAVOLAINEN,, 1999). Entretanto, o modo como cada organização desenvolve esse processo de mudança varia e depende, de certa maneira, das características culturais predominantes.

O TQM tem em seu escopo certos valores que determinam sua ideologia e essa ideologia é composta por sistemas de ideias introduzidos por vários autores ao longo de várias décadas, difundindo princípios, métodos, técnicas e ferramentas (GARVIN, 1993; SAVOLAINEN, 1997). A ideologia do TQM pode ser resumida como a melhoria contínua de produtos e processos para melhor satisfazer as necessidades dos clientes. Porém, a melhoria contínua não está colada somente à abordagem do TQM, outras abordagens emergentes têm no seu escopo também a melhoria contínua. O que se tem de diferente entre essas abordagens, tais como a do Seis Sigma e do TPM, é a existência da proposta de uma estrutura formalizada para a organização e a operacionalização das atividades de MC. E esse formalismo, em um primeiro momento top down, pode ser um elemento decisivo para o sucesso das atividades de melhoria contínua, pelo seu pragmatismo e controle das atividades de melhoria. Posteriormente, modelos bottom-up podem ser implementados, pois são menos efetivos para a melhoria contínua que um modelo top-down. Entretanto, essa é uma questão difícil pelo fato do sistema de implementação de cima para baixo ser muito centralizador, o que pode prejudicar as sugestões espontâneas.

De acordo com Boer e Gertsen (2003), as mudanças na estrutura organizacional para a melhoria contínua, ocorrida nas últimas décadas, foram respostas à crescente segmentação das demandas, às quais as empresas tiverem que elaborar respostas. Como consequência, surge a necessidade de mudanças nas estratégias competitivas e o uso de novos indicadores de desempenho. Segundo esses autores, a estratégia evoluiu do foco na eficiência (ou seja, no preço), isso na década de sessenta, para o trinômio qualidadeflexibilidade-agilidade, nas décadas de setenta a noventa. No século XXI, adota-se a inovação e aprendizado organizacional como nova estratégia competitiva, que teve como suporte os programas de melhoria contínua baseado na abordagem japonesa do Kaizen. A organização e operacionalização das empresas, para essa nova estratégia competitiva, vieram com novos programas, como o TPM, que focou a agilidade e flexibilidade de produção.

\subsection{Caracterização do TPM}

Outro programa que envolve a melhoria contínua é o TPM. Oficialmente a sigla TPM surge em 1971, cunhado pela JPE (Japan of Plant Engineering). Na época, o TPM estava restrito ao setor de produção e 
tinha as seguintes finalidades: i) maximizar a eficiência do equipamento (overall equipment efficiency); ii) estabelecer um sistema total de manutenção, projetado para a vida inteira do equipamento; e iii) operar em todos os setores envolvidos com equipamentos, incluindo setores de planejamento, operação e manutenção. O TPM tem como base a participação de todos os membros da empresa, desde a alta gerência aos membros da linha de frente e é realizado por meio de atividades em pequenos grupos (SUZUKI, 1994).

A principal evolução do TPM ocorre no final da década de oitenta, com a definição de TPM empresarial, com as seguintes características: i) criar um sistema corporativo que maximiza a eficiência do sistema de produção (overall efficiency improvement); ii) criar sistemas para impedir a ocorrência de todas as perdas na linha; e iii) estar focado no produto acabado. "Isto inclui sistemas para realizar zero acidentes, zero defeitos e zero falhas no ciclo de vida inteiro do sistema de produção (SHIROSE; BODEK, 1992)". Atualmente o TPM é aplicado em todos os setores, incluindo os departamentos de produção, desenvolvimento e administração. Outra característica do programa é propiciar a aproximação da alta gerência aos empregados da linha de frente, que desenvolvem atividades de melhoria em pequenos grupos.

O TPM pode ser considerado parte do TQM, pois juntos têm a finalidade de melhorar a estrutura das empresas e seus resultados, bem como a criação de um ambiente de trabalho agradável. Não obstante, os métodos e técnicas do TPM têm as mesmas características do TQM.

As duas abordagens podem se complementar na medida em que ambas tratam de elementos que se apoiam. Na garantia da qualidade, a ênfase é na sistematização do controle, enquanto que no TPM, a ênfase está nas condições ideais do local de trabalho e dos equipamentos, ou seja, criar um ambiente limpo e uma planta que oferece zero acidentes, zero defeitos e zero falhas. Outro aspecto é que o TPM lida com a tecnologia inerente do equipamento e com as habilidades de manutenção (SHIROSE; BODEK, 1992).

A organização formal das atividades de pequenos grupos é fortalecida no TPM, ou seja, as atividades dos pequenos grupos é parte integrante do trabalho, com a participação de todos, da gerência ao pessoal da linha de frente (nesse aspecto, caracteriza-se como um sistema bottom-up). Entretanto, não é necessário começar com o TQM antes do TPM. Caso se opte por começar pelo TPM, o foco será nas causas dos desperdícios, em que se buscará a zero perda (SHIROSE; BODEK, 1992).

O programa de implementação do TPM tradicional é constituído por 8 pilares, a saber:

- Melhoria Específica (ME);

- Manutenção Autônoma (MA);
- Manutenção Planejada (MP);

- Treinamento (TE);

- Controle de Novos Projetos (CNP);

- Manutenção da Qualidade (MQ);

- Aumento da eficiência nos setores administrativos (ADM); e

- Gestão da Segurança, Higiene e Meio Ambiente (SHM).

Uma característica do TPM é envolver desde o início o grupo em atividades de melhoria, denominadas de melhoria específica. Com isso, os resultados tendem a surgir rapidamente, motivando a participação e a obtenção do apoio da alta gerência para o projeto.

De acordo com Nakazato (1999), as empresas que implantam o TPM invariavelmente alcançam resultados de destaque. Conforme as atividades TPM começam a render resultados concretos (melhorando o local de trabalho, minimizando as quebras, melhorando a qualidade, elevando a produtividade, etc.), os trabalhadores se motivam, aumenta a integração no trabalho, e proliferam as ideias de melhorias. As pessoas começam a pensar no TPM como parte necessária do seu trabalho cotidiano. Essa postura se manifesta nos departamentos produtivos e administrativos.

Segundo Nakazato (1999), está surgindo uma nova geração do TPM, que tem como princípio, além da implementação das atividades de melhoria da eficiência global dos equipamentos, as atividades destinadas ao aumento da rentabilidade das plantas, com a participação de todos os setores e com a utilização da árvore de eficiência global. Com isso, segundo o autor, o TPM passa a ter um papel estratégico, focado em três perdas gerais, que são: a) perda no custo de fabricação; b) perda no volume de fabricação e produção; c) perda no gerenciamento de fabricação.

De acordo com a nova definição, as atividades baseadas nos 8 pilares sugerem a disseminação do TPM por toda a empresa com o objetivo de aumentar a eficiência do sistema produtivo. O TPM designado de Segunda Geração tem como princípio as atividades destinadas ao aumento da rentabilidade das plantas, com a participação de todos os setores e com a utilização das árvores de eficiência global da fábrica e de todas as perdas. Estas vêm sendo sugeridas a partir do ano de 1993, além da implementação das atividades de melhoria da eficiência global dos equipamentos ou das plantas.

Os pontos que caracterizam esta árvore são: em primeiro lugar, a identificação e melhoria de todas as perdas do volume de produção em valores monetários que correspondem à eficiência global dos equipamentos e, em segundo lugar, o acréscimo das perdas de custos de fabricação e custos de gerenciamento de fabricação com o objetivo de se destacar as vantagens (NAKAZATO, 1999). 


\section{Método de pesquisa}

\subsection{Descrição do objeto de estudo}

Neste trabalho, é realizada uma pesquisa descritiva e a técnica de coleta de dados aplicada foi um questionário estruturado, com questões fechadas (GIL, 2002). O objeto de estudo são todos os projetos de melhoria contínua de sucesso, reconhecidos por suas empresas, que tiveram como abordagem o TQM ou TPM. Como não é possível ter acesso a toda a população, foram selecionados, para a amostra, projetos de melhoria indicados pelas empresas para participarem de eventos de premiação dos melhores casos de MC ou da aplicação do TPM promovido por uma empresa de consultoria. $\mathrm{O}$ critério de escolha dos projetos baseou-se, portanto, na relevância desses para as empresas, especialmente em termos de resultados nos objetivos estratégicos. Deste modo, não foi utilizada uma amostragem probabilística, mas sim uma amostragem intencional. Entretanto, os quarenta e dois (42) projetos de melhoria contínua analisados, oriundos de várias empresas de diferentes setores e segmentos de mercado, representam a população estudada: os projetos de maior impacto nos resultados das empresas. Nem todas as empresas que fizeram parte da amostra utilizam o programa/método Seis Sigma ou implementaram a ISO 9001.

Como já mencionado, para garantir a representatividade, os projetos selecionados para estudo foram aqueles que tiveram algum reconhecimento público, primeiramente pela empresa, em seguida por representantes de outras empresas. Para isso, foram selecionados para a amostra todos os projetos indicados para participarem do evento "Casos de Excelência" promovido nos últimos cinco anos pela empresa Loss Prevention. Esse evento ocorre em São Paulo e tem participantes de empresas representantes de todas as regiões do Brasil. Nos eventos são apresentados de 30 a 50 projetos de melhoria contínua baseados no TPM e TQM. Portanto, os projetos analisados nessa pesquisa foram implementados e geraram resultados significativos para as empresas.

\subsection{Definição das variáveis de pesquisa}

$\mathrm{Na}$ análise desses projetos, levou-se em conta a organização e a operacionalização das atividades de melhoria contínua, as técnicas e ferramentas utilizadas e os resultados alcançados por esses projetos, e também o impacto no aprendizado organizacional. A seleção desses critérios de análise dos projetos baseou-se em fundamentos teóricos, em especial de Bessant (1999) e Bessant, Caffyn e Gallagher (2001). Foram selecionados para a amostra os projetos apresentados no evento Loss Prevention entre 2003 e 2007.
Ao todo, 42 projetos foram selecionados e analisados a partir de um conjunto de questões fechadas, às quais foi atribuída um escala de valor por juízes do evento. Os aspectos dos projetos que foram avaliados são as seguintes:

- Complexidade do projeto, considerando nesse julgamento o número de pessoas de diferentes áreas envolvidas no projeto, o tempo de duração e custos envolvidos;

- O problema focado, se de custos de produção, volume de produção, controle da produção, estoques, distribuição e desenvolvimento de produto;

- Os objetivos do projeto em relação aos planos estratégicos da empresa, em termos de perda de tempo, perda de desempenho e perda da qualidade;

- Técnicas e ferramentas aplicadas, tais como Pareto, 5W1H, diagrama de causa e efeito, SMED; e

- Impactos na melhoria de produtos e processos e no aprendizado organizacional.

Em cada um desses aspectos foi analisado um conjunto de variáveis com a finalidade de classificar os projetos segundo a proposta de pesquisa.

Às variáveis foram atribuídos graus de importância segundo o julgamento e percepção dos avaliadores do projeto. Como foi utilizada a pontuação segundo uma escala Likert para as variáveis em análise, tem-se, portanto, uma escala qualitativa ordinal. Para Basilevsky (1994), a escolha da escala de medição depende do fenômeno ou objeto a ser estudado, do escopo e propósito da análise e da qualidade dos dados disponíveis. As escalas ordinais e nominais são classificadas como variáveis qualitativas, sendo que em algumas situações usa-se a suposição de continuidade das escalas ordinais. O mesmo autor recomenda o uso de testes de significância não paramétricos para esse tipo de escala.

\subsection{Procedimento de análise dos dados}

Em adequação ao tipo de escala utilizada, os resultados foram analisados por técnicas descritivas e multivariadas, com testes não paramétricos, por meio das técnicas de $K$-means, cluster e discriminante. As análises foram feitas com o software Statistica ${ }^{\circledR}$ versão 9.0. Fizeram parte da pesquisa projetos realizados por empresas fabricantes de peças plásticas e de borracha (2 empresas), do setor de autopeças (4), de fundição (1), de serviços de distribuição de energia (1), do setor alimentício (2), de insumos agrícolas (1), de matérias-primas básicas (3), de tratamento térmico (1) e de higiene pessoal (1). Ao todo foram 16 empresas que apresentaram os 42 projetos de melhoria contínua nos últimos cinco anos. Todas 
as empresas são de médio e grande porte (acima de 100 funcionários), multinacionais e líderes nos setores da economia em que participam.

O uso de técnicas multivariadas, que para seu uso pressupõe dependência entre as variáveis, deve-se ao fato de que nesta pesquisa se procura compreender aspectos subliminares nas relações entre constructos (BASILEVSKY, 1994). Classificadas na categoria de técnicas multivariadas, recomendadas em análises exploratórias, caso desta pesquisa, estão, dentre outras, as análises de Cluster, Fatorial, Discriminante, de Correspondência e de Confiabilidade dos instrumentos de pesquisa. Os autores deste trabalho optaram pelo uso das análises de Correspondência e de Cluster. Fez-se uso de testes não paramétricos, que devem ser aplicados quando a qualidade dos dados não é boa; por exemplo, quando o tamanho da amostra é pequeno e/ou a distribuição dos dados é desconhecida. Testes não paramétricos comumente aplicados são os de Wald-Wolfowitz runs test, o Mann-Whitney $U$ test, e o Kolmogorov-Smirnov two-sample test. Para múltiplos grupos, o teste paramétrico geralmente aplicado é baseado na análise de variância (ANOVA/ MANOVA). O equivalente não paramétrico são os testes Kruskal-Wallis. Maiores detalhes sobre essas técnicas podem ser obtidos na literatura (SIEGEL; CASTELLAN, 1988; JOBSON, 1991; GARCIA, 1995; MONTGOMERY; RUNGER, 2003).

\subsection{Validação do instrumento de pesquisa}

O conceito se confiabilidade denotado nas pesquisas de ciências sociais é diferente da ideia de confiabilidade na estatística industrial. A confiabilidade de um resultado empírico, tais como a relação entre variáveis encontradas em uma amostra, depende da representatividade da amostra em relação a uma população específica. Isso implica na reprodutibilidade da pesquisa e significa interesse não na amostra, mas na medida em que a amostra traz informações sobre a população. Na presente pesquisa, o estudo conduzido nas etapas finais do projeto pretende estabelecer medidas estatísticas da relação entre variáveis obtidas por meio de testes de significância, tecnicamente chamados de $p$-valor ou significância estatística. A confiabilidade dos instrumentos de medidas que analisa hipóteses de um processo que envolve julgamentos e crenças já é em si uma pesquisa, pois há muitas variáveis difíceis de serem medidas. Estudos da confiabilidade dos instrumentos de pesquisa no contexto abordado são recomendados na análise da melhoria do instrumento de pesquisa em desenvolvimento e na pesquisa em curso para avaliar a confiabilidade das respostas (HAIR JUNIOR et al., 2005).

Deste modo, a avaliação da confiabilidade da escala é uma medida relativa entre a correlação dos itens individualmente e a variância dos itens. Para avaliar a confiabilidade de uma escala de medida é utilizada comumente o Alpha de Cronbach, cuja fórmula é indicada a seguir (Equação 1):

$$
\alpha=(k /(k-1)) *\left[1-\left(s_{i}^{2}\right) / s_{\text {soma }}^{2}\right]
$$

A variância de cada item $k$ é estimada por $s^{2}{ }_{i} \mathrm{e}$ $s_{\text {soma }}^{2}$ denota a soma da variância de todos os itens. Se a soma da variância de cada item for igual à variância da soma de todos os itens a confiabilidade é zero. Caso a soma da variância de cada item for muito menor que a variância total o $\alpha$ será próximo de 1. Portanto, quando maior o Alpha de Cronbach melhor é a confiabilidade da escala para avaliar o julgamento dos indivíduos. Quando o contrário ocorre, indica uma grande parcela de subjetividade no item avaliado. Assim, quanto mais itens são colocados para avaliar um conceito, mais confiável será o instrumento de pesquisa.

Teorias para o desenvolvimento de escalas de medidas em pesquisa nas ciências sociais e econômicas podem ser visto em Carmines e Zeller (1980) e Gruitjer e van der Kamp (1976).

Deste modo, a validação do instrumento de coleta de dados foi feita por meio do Alpha de Cronbach, que para o questionário aplicado foi de 0,78 e o recomendado na literatura como mínimo é de 0,70 (HAIR JUNIOR et al., 2005).

\section{Resultados e análises}

\subsection{Relação entre a complexidade dos projetos e seus objetivos}

Os projetos analisados tiverem três objetivos principais, mostrados na Tabela 1, que focaram as perdas de tempo, perdas de desempenho e perdas de qualidade. Foi atribuído aos projetos um grau de complexidade que levou em conta, como variáveis, o número de pessoas de diferentes áreas envolvidas no projeto, o seu tempo de duração e custos envolvidos. A análise de correlação entre a complexidade dos projetos com os seus objetivos relaciona as três perdas gerais estratégicas segundo a abordagem do TPM. A relação entre duas variáveis aleatórias $X$ e $Y$ é geralmente caracterizada pela covariância ou pelo coeficiente de correlação, $p$. O coeficiente de correlação de Pearson, em geral utilizado para variáveis quantitativas, mede a associação linear entre duas variáveis. Para variáveis qualitativas ordinais, que é o caso desta pesquisa, o coeficiente de correlação recomendado é o de Spearman, que, ao invés do produto momento, usa o ordenamento separado das variáveis para determinar a relação entre elas (JOBSON, 1991). Os projetos foram classificados em três níveis de complexidade: 3-alta complexidade, 2-média complexidade, 1-baixa complexidade. A complexidade foi relacionada aos 
objetivos do projeto e não foi identificada correlação entre a complexidade dos projetos com as três perdas que compreende a abordagem do TPM.

Duas conclusões podem ser extraídas dessa análise, uma é que não há um foco específico dos objetivos dos projetos, por exemplo, para a melhoria da qualidade ou perda de tempo; e, em segundo lugar, que a complexidade do projeto não está relacionada ao seu objetivo. Assim, não há evidência de que projetos para a melhoria da qualidade sejam mais ou menos complexos que projetos para a redução de perda de tempo da produção. O que se verifica é que projetos relacionados a perdas de tempo na produção estão mais relacionados a perdas de desempenho, quando esta é comparada à perda de qualidade. Isto pode indicar que priorizar as perdas de tempo gera melhor desempenho se comparado ao objetivo de perda de qualidade. O que se conclui é que o foco na redução de perda de tempo tem gerado mais resultados que o foco nas perdas da qualidade. Disto decorre que a qualidade não é o principal objetivo da maioria dos projetos analisados.

\subsection{Relação entre a complexidade do projeto e a estratégia da empresa}

A análise da formação dos grupos, do tempo de execução dos projetos e custos envolvidos permitiu concluir que grupos, aos quais foram atribuídos projetos estratégicos, possuem uma estrutura mais complexa, envolvendo pessoas de diferentes áreas, mais recursos financeiros e com uma maior duração da sua execução. Isso está de acordo com as novas abordagens da MC, voltadas para projetos mais estratégicos, vistas na revisão bibliográfica.
A Tabela 2 mostra a relação entre a complexidade dos projetos e a relação dessa variável com a orientação estratégica dos projetos atribuídos a esses grupos. Obteve-se forte correlação $(0,70)$ entre a complexidade dos projetos e a relação com a estratégia da empresa. Esse resultado é corroborado pela análise de cluster apresentada na Figura 2. Verifica-se que projetos estratégicos demandam estruturas de grupos com maior interação lateral, ou seja, envolvem mais departamentos e setores da empresa. Diferentemente, projetos menos estratégicos levam um tempo menor para serem concluídos e utilizam menos pessoal técnico e de outros setores e departamentos da empresa. Há indícios, indicados na Tabela 2 , de que há relação entre aprendizado e melhoria contínua.

\subsection{Relação entre melhoria e aprendizado}

Na Figura 1 é mostrada a análise de cluster aplicada às variáveis estudadas que indica relação entre melhoria e aprendizado. Como sugere a literatura, atividades de melhoria contínua contribuem para o aprendizado organizacional por meio das atividades de grupo. Semelhante à análise de cluster, é a análise de correspondência, mostrada na Figura 2 que indica que projetos mais complexos são mais relacionados à estratégia. A questão é se projetos de melhorias incrementais menos estratégicos levam ao aprendizado organizacional na mesma magnitude que os projetos estratégicos.

Foram formados três clusters de empresas, com base nas variáveis de pesquisa. As médias atribuídas a cada cluster são mostradas na Tabela 3 , sendo que o cluster 2 foi o que teve menores escores médios e os clusters 1 e 3 tiverem escores médios maiores. As médias de cada variável segmentada por cada cluster são apresentadas no Figura 3. As variáveis que mais

Tabela 1. Relação entre complexidade e objetivos dos projetos.

\begin{tabular}{lccc}
\hline & \multicolumn{3}{c}{ Objetivos do projeto } \\
\cline { 2 - 4 } & Perda tempo & Perda desempenho & Perda qualidade \\
\hline Complexidade & 0,256 & 0,115 & 0,260 \\
Perda tempo & 1,000 & $-0,323^{*}$ & $-0,287$ \\
Perda desempenho & - & 1,000 & 0,269 \\
Perda qualidade & - & - & 1,000 \\
\hline
\end{tabular}

*correlação com nível de significância de 5\%.

Tabela 2. Relação da complexidade dos projetos com a estratégia da empresa.

\begin{tabular}{lcccc}
\hline \multicolumn{1}{c}{ Variáveis } & $\begin{array}{c}\text { Correlação } \\
\text { estratégia }\end{array}$ & $\begin{array}{c}\text { Impacto na } \\
\text { estratégia }\end{array}$ & $\begin{array}{c}\text { Impacto na } \\
\text { melhoria }\end{array}$ & $\begin{array}{c}\text { Grau } \\
\text { aprendizado }\end{array}$ \\
\hline Complexidade & $0,738^{*}$ & 0,506 & 0,316 & 0,475 \\
Correlação estratégia & 1,000 & 0,462 & 0,070 & 0,439 \\
Impacto na estratégia & - & 1,000 & 0,418 & 0,316 \\
Impacto na melhoria & - & - & 1,000 & 0,486 \\
Grau aprendizado & - & - & - & 1,000 \\
\hline
\end{tabular}

*correlação com nível de significância de $1 \%$. 


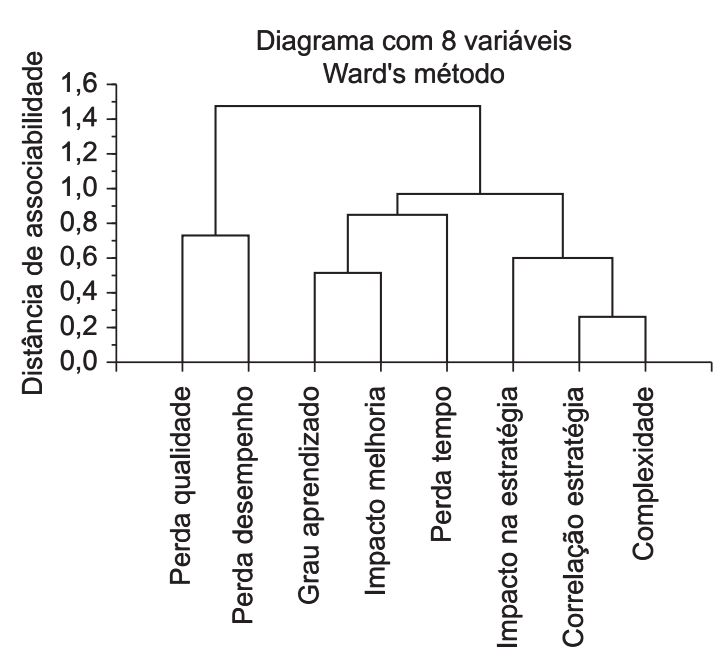

Figura 1. Relação entre aprendizado e melhoria.

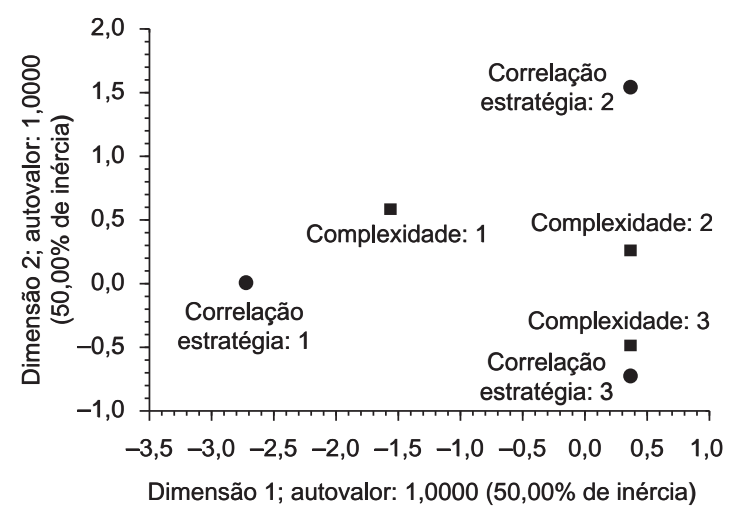

- Coordenadas das colunas principais

- Coordenadas das colunas suplementares

Figura 2. Correspondência entre complexidade do projeto e estratégia.

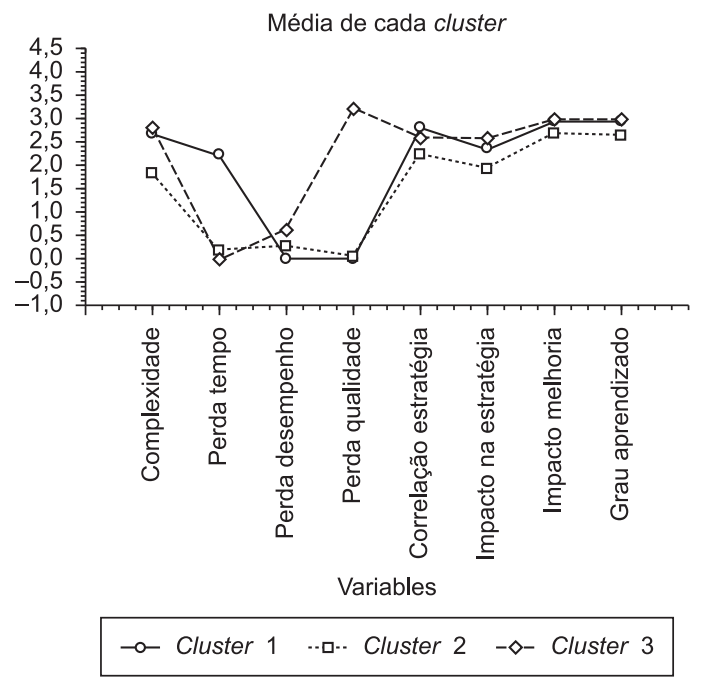

Figura 3. Formação dos clusters e médias das variáveis. influenciaram na formação dos clusters foram as variáveis perda de tempo, perda de qualidade e de complexidade dos projetos. A Tabela 4 mostra os níveis de significância de cada variável na formação dos clusters - observa-se que os níveis de significância para as variáveis perda de tempo, perda de qualidade e de complexidade são inferiores a 0,0001. A variável que menos contribuiu na formação dos clusters foi impacto na melhoria.

Dos quarenta e dois (42) projetos analisados, cinco (5) compuseram o cluster 3 , quinze (15) projetos fizeram parte do cluster 1 e vinte e dois (22) compuseram o cluster 2. Como uma das variáveis principais de dissimilaridade entre o cluster 1 e 3 foi Perda de Qualidade, o cluster 3 teve todos os seus projetos relacionados à qualidade de conformação teve dois projetos de redução de defeitos de qualidade, um projeto focado na redução de custos, um focado no aumento da produção e um projeto focado na redução de resíduos industriais. Isso significa que $12 \%$ dos projetos focaram a perda de qualidade de conformação.

O cluster 1 teve todos os seus projetos relacionados à perda de tempo na produção: oito abordaram a manutenção de equipamentos de produção; três focaram a redução de setup; e os demais trataram do aumento da produtividade, do aumento da capacidade produtiva e da influência do meio ambiente na manutenção dos equipamento. Isso representa $36 \%$ dos projetos, bem maior que os $12 \%$ que trataram da qualidade de conformação. Vê-se que os projetos de melhoria têm focado mais a manutenção dos equipamentos e perdas de tempo na produção, isso se justifica pela adoção por parte das empresas da abordagem do TPM.

Os projetos do cluster 2 (53\% da amostra) têm focado redução de ciclo de produção, tempo de alimentação de máquina, desperdícios de materiais, consumo de água, disponibilidade de máquinas. Esses projetos foram considerados menos complexos que os projetos dos associados aos clusters 1 e 3 .

Cabe destacar que as empresas que fizeram parte dos clusters 1 e 3 são unicamente indústrias manufatureiras, de produção contínua e de processo

Tabela 3. Formação de clusters de empresas e suas dissimilaridades.

\begin{tabular}{lccc}
\hline \multicolumn{1}{c}{ Variáveis } & Cluster 1 & Cluster 2 & Cluster 3 \\
\hline Complexidade & 2,667 & 1,818 & 2,800 \\
Perda tempo & 2,200 & 0,182 & 0,000 \\
Perda desempenho & 0,000 & 0,273 & 0,600 \\
Perda qualidade & 0,000 & 0,045 & 3,200 \\
Correlação estratégia & 2,800 & 2,227 & 2,600 \\
Impacto na estratégia & 2,333 & 1,909 & 2,600 \\
Impacto da melhoria & 2,933 & 2,682 & 3,000 \\
Grau aprendizado & 2,933 & 2,636 & 3,000 \\
\hline
\end{tabular}


repetitivo dos setores da indústria de plásticos e de metal mecânica do segmento automobilístico. Já as empresas do cluster 2 são de diferentes setores que compreendem, em especial, os setores de serviços e saneamento básico. Outro aspecto observado é que os pilares: Controle de Novos Projetos (CNP), Manutenção da Qualidade (MQ), Aumento da eficiência nos setores administrativos (ADM) e Gestão da Segurança, Higiene e Meio Ambiente (SHM) não são ainda projetos centrais das empresas que adotam o TPM. Isso é reforçado quando se observa a Figura 6: as empresas, por meio dos seus projetos, têm adotado mais os pilares MA e ME. O pilar MQ tem pouco destaque nos projetos do TPM.

\subsection{Relação entre aprendizado, técnicas e estratégias}

Quanto ao uso das técnicas/ferramentas, o que se observa é a aplicação de ferramentas mais qualitativas, como por exemplo, o $5 \mathrm{~W} 1 \mathrm{H}$, útil no planejamento de ações. Relacionados a ela aparecem a análise de Pareto, útil na identificação de prioridades, e o diagrama de causa e efeito, utilizado na análise das

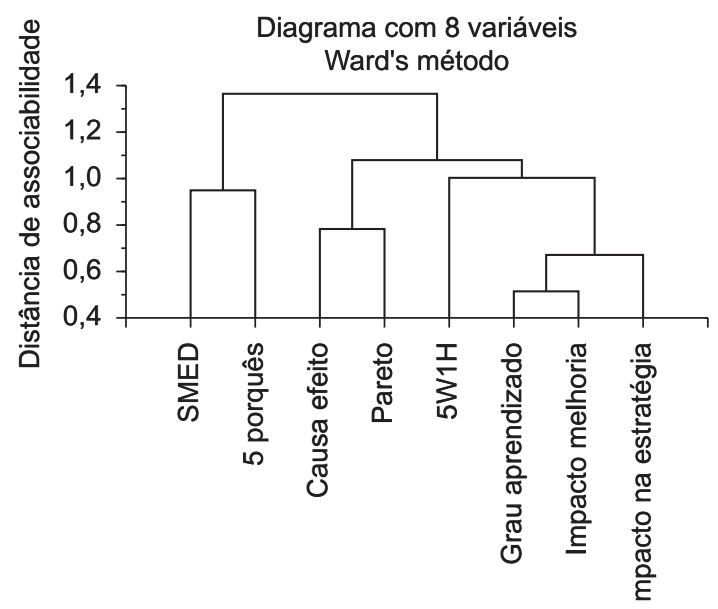

Figura 4. Relação entre aprendizado, ferramentas e estratégia. causas dos problemas, como está indicado na Figura 4. A ferramenta $5 \mathrm{~W} 1 \mathrm{H}$ é uma ferramenta adequada para que se estabeleçam vínculos entre o projeto e a estratégia da empresa. Isso pode em parte explicar a relação dessa técnica com os objetivos estratégicos da empresa mostrada na análise da Figura 2.

A Figura 5 mostra a relação entre a complexidade dos projetos, impacto na melhoria, grau de aprendizado e impacto na estratégia. Percebe-se que projetos mais estratégicos têm correspondência ao aprendizado organizacional e tem maior impacto na melhoria contínua. Há duas implicações: primeiro, um projeto complexo condiz com a adoção de programas de gestão estruturados para a melhoria contínua, com governança e apoio às atividades do projeto pela alta administração; segundo, indica que uma abordagem estratégica dos projetos condiciona o aprendizado organizacional.

\subsection{Relação entre os projetos e pilares do TPM}

A Figura 6 constata que a complexidade do projeto está relacionada aos pilares do TPM da Manutenção

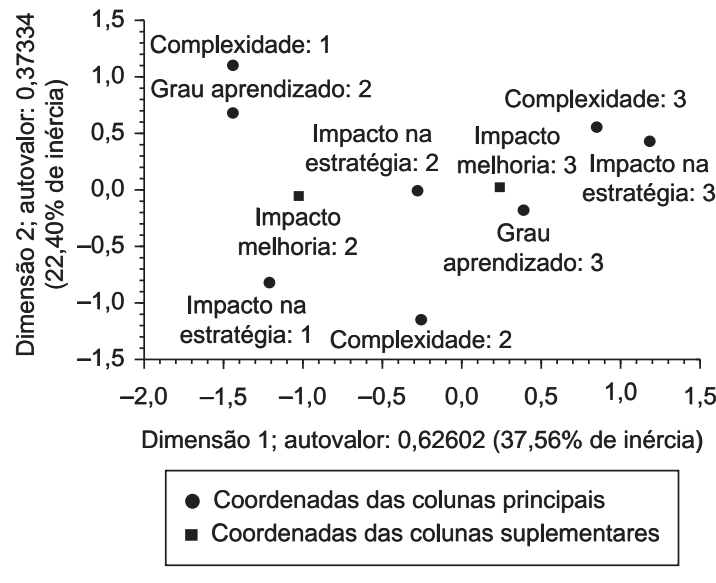

Figura 5. Correspondência entre melhoria, estratégia e aprendizado.

Tabela 4. Análise de Variância das variáveis que formaram os clusters.

\begin{tabular}{lcccccc}
\hline \multicolumn{1}{c}{ Variáveis } & $\begin{array}{c}\text { Variação } \\
\text { entre as } \\
\text { variáveis }\end{array}$ & $\begin{array}{c}\text { Graus de } \\
\text { liberdade das } \\
\text { variáveis }\end{array}$ & $\begin{array}{c}\text { Variação } \\
\text { interna }\end{array}$ & $\begin{array}{c}\text { Graus de } \\
\text { liberdade } \\
\text { dos resíduos }\end{array}$ & F & $\begin{array}{c}\text { P - nível de } \\
\text { significância }\end{array}$ \\
\hline Complexidade & 8,213 & 2,000 & 15,406 & 39,000 & 10,395 & 0,000 \\
Perda tempo & 40,732 & 2,000 & 13,673 & 39,000 & 58,092 & 0,000 \\
Perda desempenho & 1,508 & 2,000 & 9,564 & 39,000 & 3,074 & 0,058 \\
Perda qualidade & 44,365 & 2,000 & 5,755 & 39,000 & 150,335 & 0,000 \\
Correlação estratégia & 3,013 & 2,000 & 17,464 & 39,000 & 3,364 & 0,045 \\
Impacto na estratégia & 2,791 & 2,000 & 12,352 & 39,000 & 4,407 & 0,019 \\
Impacto melhoria & 0,770 & 2,000 & 5,706 & 39,000 & 2,632 & 0,085 \\
Grau aprendizado & 1,047 & 2,000 & 6,024 & 39,000 & 3,390 & 0,044 \\
\hline
\end{tabular}


Autônoma (MA) e à Manutenção Específica (ME), ao passo que a Manutenção da Qualidade (MQ) fica classificada fora deste grupo.

Corroborando com essa análise da Figura 6, a Figura 7 mostra a relação entre os pilares do TPM com o foco nas perdas de tempo e da qualidade, indica associação entre o foco na perda de tempo com os pilares da Manutenção Planejada (MP), Manutenção Específica (ME) e Manutenção Autônoma (MA). A perda em qualidade está fora do foco principal dos projetos de melhoria que trabalham com a abordagem

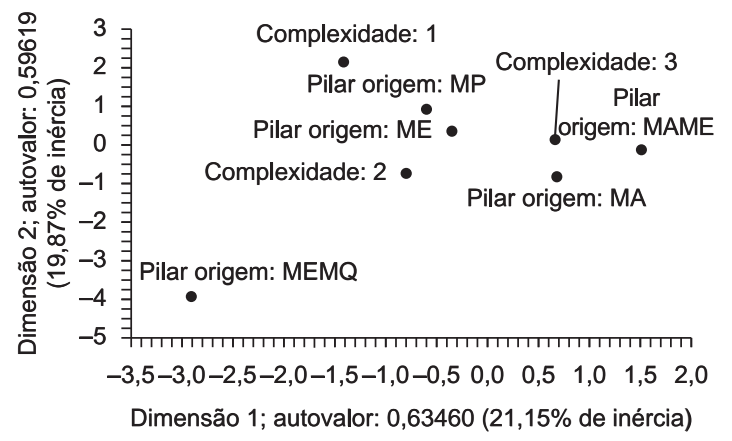

Figura 6. Correspondência entre os pilares do TPM e complexidade dos projetos.

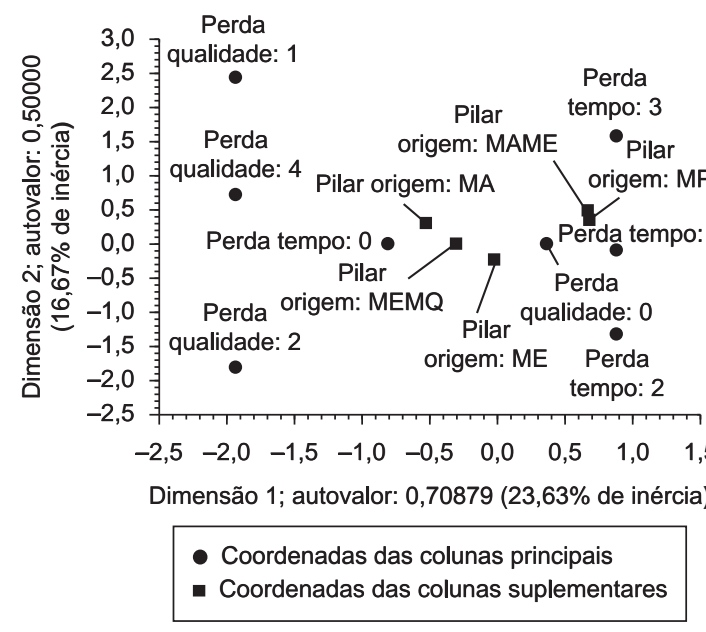

Figura 7. Correspondência entre os pilares do TPM, perda de tempo e perda da qualidade. do TPM. Comprovando a análise das Figuras 5 e 6 , a Tabela 5 mostra que, de todos os projetos, o pilar de Manutenção Específica é o que mais aparece nos projetos. O pilar MQ aparece pouco nos projetos analisados.

\subsection{Análises dos resultados}

Com base nas análises da amostra estudada, que se caracteriza basicamente por empresas manufatureiras, verifica-se que, apesar da teoria do TPM e do TQM indicarem outras dimensões e objetivos voltados a questões sociais e ambientais, o foco principal dos projetos é ainda a eficiência da produção, pela redução do tempo, ciclos e de custo de produção. No TPM são sustentados pelos pilares de origem dos projetos da Manutenção Específica (ME) e da Manutenção Autônoma (MA).

O que se percebe é que, os objetivos estratégicos da produção continua sendo a eficiência operacional, isso significa reduzir os custos de produção. Conclui-se que o TPM, apesar dos novos paradigmas de produção decorrentes do Sistema Toyota de Produção, tem a mesma preocupação do Fordismo/Taylorismo, a da eficiência da fábrica, com objetivos um pouco mais complexos, que são os seguintes: redução de setup, de ciclo de produção, de perdas de materiais, para reduzir tempo e aumentar a produção.

Quanto à organização e operacionalização do processo de melhoria contínua, verifica-se a formação de grupos com pessoas provenientes de vários setores em projetos mais complexos. Isso é exigido quando os projetos demandam conhecimentos técnicos que o grupo do local de trabalho não tem e que são necessários para resolverem os problemas do projeto. Esse aspecto é sustentado pela revisão teórica apresentada neste trabalho. Entretanto, vê-se a importância da participação do pessoal diretamente ligado à produção, compartilhando conhecimentos e experiências, propiciadas por abordagens do tipo do TPM.

\section{Conclusões}

Este trabalho mostra que os projetos de melhoria contínua, desenvolvidos por empresas que adotam a abordagem do TQM/TPM e considerados mais complexos, estão relacionados a objetivos estratégicos

Tabela 5. Relação entre os projetos e pilares de origem do TPM.

\begin{tabular}{lccccc}
\hline Complexidade & $\begin{array}{c}\text { MA: pilar } \\
\text { Manutenção } \\
\text { Autônoma }\end{array}$ & $\begin{array}{c}\text { ME: pilar } \\
\text { Manutenção } \\
\text { Específica }\end{array}$ & $\begin{array}{c}\text { MP: pilar } \\
\text { Manutenção } \\
\text { Planejada }\end{array}$ & $\begin{array}{c}\text { MQ e ME: } \\
\text { Manutenção da } \\
\text { Qualidade e Específica }\end{array}$ & $\begin{array}{c}\text { MA e ME: } \\
\text { Manutenção } \\
\text { Autônoma e Específica }\end{array}$ \\
\hline 1 & 0 & 7 & 1 & 0 & 0 \\
2 & 3 & 10 & 1 & 1 & 1 \\
3 & 4 & 10 & 1 & 0 & 3 \\
Total & 7 & 27 & 3 & 1 & 4 \\
\hline
\end{tabular}


e que esses geram maior impacto nos resultados da empresa. Com base nos resultados empíricos, pode-se dizer que os objetivos estratégicos da produção vigentes incorporou aos indicadores clássicos de produtividade, obtidos por ganhos de escala de produção, a flexibilidade e agilidade, por meio da redução dos tempos mortos de produção. $\mathrm{O}$ que está de acordo com as análises de Boer e Gertsen (2003) sobre a evolução das abordagens estratégias e a relação destas com a inovação/melhoria contínua e aprendizagem organizacional.

Esse resultado é evidenciado quando se analisam os objetivos dos projetos. O objetivo principal dos projetos foi a agilidade e flexibilidade da produção, focados na manutenção de equipamentos e redução de setups. Já o número de projetos focados nas perdas de qualidade na fabricação, além de serem considerados menos estratégicos na avaliação dos projetos, teve, em números absolutos, menor quantidade que os focados na agilidade/tempo e flexibilidade da produção. Isso indica que as empresas têm no tempo/ agilidade e na flexibilidade seus principais objetivos de eficiência operacional. Outros temas de grupos de melhoria, como perdas de materiais, impacto no meio ambiente e segurança no trabalho, não foram temas secundários dos grupos/projetos analisados considerados estratégicos.

Apesar das limitações em termos de generalização dos resultados dessa pesquisa, é possível extrapolar as conclusões apresentadas para modelos orgânicos de estruturação das organizações para a MC.

A partir desses resultados, pode-se indicar que os projetos considerados mais complexos geraram maior aprendizado organizacional e maior impacto nos resultados das empresas. Entretanto, não se perceberam as mesmas relações com projetos voltados à melhoria da qualidade do produto no cliente, pois os maiores esforços das empresas estão na redução das perdas de tempo de produção para obter maior flexibilidade e agilidade. Essas conclusões são fortalecidas quando se analisam os pilares do TPM, pois os dados indicam que a prioridade na adoção dessa abordagem/programa é a manutenção específica e a manutenção autônoma; esses pilares foram considerados mais estratégicos que os demais pilares.

Quanto ao uso das ferramentas, o que se observa é a aplicação de ferramentas mais qualitativas no planejamento de ações, tais como Pareto e 5W1H, que são adequadas para que se estabeleçam vínculos entre o projeto e a estratégia da empresa.

A pergunta e a proposição formuladas nos objetivos deste trabalho foram respondidas, pois se constatou que a melhoria contínua tem evoluído e contribuído para os novos objetivos estratégicos da produção e para o aprendizado organizacional. A afirmação que se confirma é que as atividades de melhoria contínua tornaram-se ao longo das últimas décadas um elemento estratégico para as empresas, à medida que, conforme Boqer e Gertsen (2003) citam, as estratégias de produção foram mudando o seu foco, da eficiência para a inovação, passando pela agilidade e flexibilidade. O que se constatou é que houve a incorporação de novos conceitos à tradicional abordagem de $\mathrm{MC}$, com formação de equipes multifuncionais, eventualmente incluindo clientes e fornecedores, no trato de questões consideradas estratégicas para as empresas. As análises indicaram evidência estatística que confirma o foco dos projetos na redução de tempos perdidos (tempos mortos) e a relação destes com a perda de desempenho. O que se verifica é que projetos relacionados a perdas de tempo na produção estão mais relacionados a perdas de desempenho, quando esta é comparada à perda de qualidade. Ou seja, perda de tempo é mais importante/estratégico que os objetivos de qualidade.

Evidências estatísticas indicam que projetos estratégicos demandam estruturas de grupos com maior interação lateral, ou seja, envolve mais departamentos e setores da empresa. Diferentemente, projetos menos estratégicos levam um tempo menor para serem concluídos e utilizam menos pessoal técnico e de outros setores e departamentos da empresa. Outra evidência estatística é a relação entre aprendizado e melhoria contínua. Projetos mais complexos são mais inovativos e conduzem a um maior aprendizado organizacional.

Como proposta de novos trabalhos de pesquisa, seria plausível estudar projetos Seis Sigma com a mesma ótica da análise feita para o TQM/TPM, para avaliar qual o enfoque principal dado pelas empresas que adotam essa abordagem. Outro fato a constatar é como são usados os métodos estatísticos nos projetos de MC.

A limitação deste trabalho é a generalização dos resultados, pois os projetos selecionados para a amostra baseou-se na indicação das empresas, por serem, segundo elas, os melhores projetos de melhoria realizados nos últimos anos.

Verificou-se também a utilidade da aplicação de técnicas da análise multivariada em pesquisas qualitativas, como foi feito neste trabalho, pois permitiu, além da validação do instrumento de pesquisa, identificar aspectos subliminares não detectáveis por análise textual ou estatística paramétrica. Por exemplo, as análises de correspondência aplicadas permitiram verificar relações entre os objetivos dos projetos, sua complexidade e o impacto desses na estratégia competitiva da produção. Tais resultados seriam de difícil observação para dados qualitativos.

\section{Referências}

ABRAHAMSSON, G; GERDIN, J. Exploiting institutional contradictions: the role of management accounting in continuous improvement implementation, Qualitative 
Research in Accounting \& Management, v. 3, n. 2, p. 126-144, 2006.

BASILEVSKY, A. Statistical factor analysis and related methods: theory and applications. New York: Wiley Interscience, 1994.

BECKET, A. J.; WAINWRIGHT, C. E. R.; BANCE, D. Implementing an industrial continuous improvement sustems: a knowledge management case study. Industrial Management \& Data Systems, v. 100, n. 7, p. 330-338, 2000.

BERGQUIST, B.; FREDRIKSSON, M.; SVENSSON, M. TQM: terrific quality marvel or tragic quality malpractice. The TQM Magazine, v. 17, n. 4, p. 309-21, 2005.

BESSANT, J. Developing continuous improvement capability. International Journal of Innovation Management, v. 2, n. 4, p. 409-429, 1999.

BESSANT, J. et al. Rediscovering continuous improvement. Technovation, v. 14, n. 1, p. 17-29, 1994.

BESSANT, J.; CAFFYN, S. High involvement innovation through continuous improvement. International Journal of Techology and Management, v. 14, n. 1, p. 7-28, 1997.

BESSANT, J.; CAFFYN, S.; GALLAGHER, M. An evolutionary model of continuous improvement behavior. Technovation, v. 21, n. 2, p. 67-77, 2001.

BESSANT, J.; FRANCIS, D. Developing strategic continuous improvement capability. International Journal of Operation \& Production Management, v. 19, n. 11, p. 1106-1119, 1999.

BHUIYAN, N.; BAGHEL, A. An overview of continuous improvement: from the past to the present. Management Decision, v. 43, n. 5, 2005.

BOER, H.; GERTSEN, F. The continuous improvement to continuous innovation: a retro perspective. International Journal Technology Management, v. 26, n. 8, p. 805-827, 2003.

BOND, T. C. The role of performance measurement in continuous improvement. International Journal Operation \& Production Management, v. 19, n. 12, p. 1318-1334, 1999.

CARMINES, E. G.; ZELLER, R. A. Reliability and validity assessment. Beverly Hills: Sage, 1980.

CHAPMAN, R. L.; HYLAND, P. Continuous improvement strategies across selected Australian manufacturing sectors. Benchmarking for Quality Management \& Technology, v. 4, n 1, p. 175-188, 1997.

CHASE, R. B.; JACOBS, F. R.; AQUILANO, N. J. Operations management for competitive advantage. 11. ed. New York: McGraw-Hill, 2006.

CUSUMANO, M. A. The limits of lean. Sloan Management Review, p. 27-32, Summer 1994.

DABHILKAR, M.; BENGTSSON, L. Balanced scorcards for strategic and sustainnable continuous improvement capability. Journal of Manufacturing Techonology Management, v. 15, n. 4, p. 350-359, 2004.

DELBRIDGE, R.; BARTON, H. Organizing for continuous improvement: structures and roles in auto components plants. International Journal of Operations and Production Management, v. 22, n. 6, p. 680-692, 2002.
EHIGIE, B. O.; McANDREW, E. B. Innovation, diffusion and adoption of total quality management. Management Decision, v. 43, n. 6, p. 925-940, 2005.

FOSTER, D.; JONKER, J. Towards a third generation of quality management. The International Journal of Managament Science, v. 24, n. 7, p. 683-703, 2007.

GARCIA, J. Análise de la información mercadológica a través de la estadística multivariante. Ciudad de Mexico: Alhambra Mexicana, 1995.

GARVIN, D. Building a learning organization. Harvard Business Review, p. 78-91, July/Aug. 1993.

GIL, A. C. Como elaborar projetos de pesquisa. 4. ed. São Paulo: Atlas, 2002.

Gruitjer, P. N. M.; van der Kamp, L. J. T. Advances in psychological and educational measurement. New York: Wiley, 1976.

HAIR JUNIOR., J. F. et al. Análise multivariada de dados. 5. ed. Porto Alegre: Bookman, 2005.

HAMMERSLEY, G.; PINNGTON, A. Emploee response to continuous improvement groups. The TQM Magazine, v. 11, n. 1, p. 29-34, 1999.

HARRINGTON, H. J. Continuous versus breakthrough improvement: finding the right answer. Business Process Re-engineering \& Management Journal, v. 1, n. 3, p. 31-45, 1995.

HYLAND, P. et al. A comparison of Australian firms and their use of continuous improvement tools. The TQM Magazine, v. 12, n. 2, p. 117-124, 2000.

IMAI, M. Gemba Kaisen: a common sense, low-cost approach to management. New York: McGraw-Hill, 1997.

JABNOUN, N. Value underlying continuous improvement. The TQM Magazine, v. 13, n. 6, p. 381-387, 2001.

JHA, S.; NOORI, H.; MICHELA, J. L. The dynamics of continuous improvement. International Journal of Quality Science, v. 1, n. 1, p. 19-47, 1996.

JOBSON, J. D. Applied multivariate data analysis: regression and experimental design. New York: Springer, 1991. v. 1.

JOINER, T. A. Total quality management and performance: the role of organization support and co-worker support. International Journal of Quality \& Reliability Management, v. 24, n. 6, p. 617-627, 2007.

JURAN, J.; GRYNA, F. Controle da qualidade. São Paulo: Makron Books; New York: Mcgraw-Hill, 1991.

LEE, H. J. The role competence-based trust and organizational identification in continuous improvement. Journal of Managerial Psychology, v. 19, n. 6, p. 623-639, 2004.

LEONARD, D.; McADAM, R. An evaluative framework for TQM dynamics in organizations. International Journal of Operations \& Production Management, v. 23, n. 6, p. 652-677, 2003.

McADAM, R.; HENDERSON, J. Influencing the future of TQM: internal and external driving factors. International Journal of Quality \& Reliability Management, v. 21, n. 1, p. 51-71, 2004.

MERLI, G. Eurochallenge: the TQM approach to capturing global markets. UK: IFS, 1993.

MONTGOMERY, C. D.; RUNGER, G. C. Estatística aplicada e probabilidade para engenheiros. 2. ed. São Paulo: LTC, 2003. 
NAKAZATO, K. Conceitos básicos do TPM. Parte II. Japão: Japan Institute of Plant Maintenance: 1999.

NILSSON-WITELL, L.; ANTONI, M.; DAHLGAARD, J. J. Continuous improvement in product development. International Journal of Quality \& Reliability Management, v. 22, n. 8, p. 753-768, 2005.

PRAJOGO, D. I.; SOHAL, A. S. Relationship between organization strategy, total quality management (TQM), and organization performance: the mediating role of TQM. European Journal of Operational Research, v. 168 , p. 35-50, 2004.

PRAJOGO, D. I.; SOHAL, A. S. TQM and innovation: a literature review and research framework. Technovation, v. 21, n. 10, p. 532-558, 2001.

RAHMAN, S.; BULLOCK, P. Soft TQM, hard TQM, and organizational performance relationships: an empirical investigation. OMEGA The international Journal of Managament Science, v. 33, n. 1, p. 73-83, 2005.

RAISINGHANI, M. et al. Six Sigma: concepts, tools, and applications. Industrial Management \& Data Systems, v. 105, n. 4, p. 491-505, 2005.

SACCHETTI, L. ISO Quality as a driver of continuous improvement. Perfomance Measurement and Metrics, v. 8, n. 2, p. 88-97, 2007.

SAVOLAINEN, T. Development of quality-oriented management ideology. Periódico, volume, n. 37, 1997.
SAVOLAINEN, T. I. Cycles of continuous improvement: realizing competitive advantage trough quality. Internationational Journal of Operations \& Production Management, v. 19, n. 11, p. 1203-1222, 1999.

SHIROSE, K; BODEK, N. TPM for workshop leaders. 1. ed. New York: Productivity, 1992.

SIEGEL, S.; CASTELLAN, N. J. Nonparametric statistics. 2. ed. New York: McGraw-Hill, 1988.

SLACK, N. et al. Administração da Produção. 2. ed. São Paulo: Atlas, 2007.

SUPANVANIJ, J.; AMINE, L. S. Consumer perception of country-of-origin effect and $\mathrm{Br}$ and effect. Latin American Business Review, v. 1, n. 4, p. 47-60, 2000.

SUZUKI, T. TPM in process industries. Portland: Productivity, 1994.

TERZIOVSKI, M. Achieving performance through an integrated strategy of radical innovation and continuous improvement. Business Excellence, v. 6, n. 2, p. 5-24, 2002.

TERZIOVSKI, M.; POWER, D. Increasing ISO 9000 Certification benefits: a continuous improvement approach. International Journal Quality \& Reliability Management, v. 24, n. 2, p. 141-163, 2007.

TOLEDO, J. C. Qualidade industrial: conceitos, sistemas e estratégias. São Paulo: Atlas, 1987.

UPTON, D. M. Designing, managing, and improving operations. New Jersey: Prentice Hall. 1998. 